www.jmscr.igmpublication.org

Index Copernicus Value: 79.54

ISSN (e)-2347-176x ISSN (p) 2455-0450

crossref DOI: https://dx.doi.org/10.18535/jmscr/v7i4.06

\title{
Feasibility of transatrial-transpulmonary total correction of TOF
}

\author{
Authors \\ Dr Nasir U Din Wani ${ }^{1}$, Dr Syed Abir Hussain ${ }^{2}$, Dr Tasneem Muzaffar ${ }^{3}$, \\ Dr Abdul Gani Ahangar ${ }^{4}$, Dr Ghulam Nabi Lone ${ }^{5}$ \\ ${ }^{1,2,3}$ Consultant CVTS Surgeon, GMC, Srinagar \\ ${ }^{4}$ Prof. Department of CVTS, SKIMS, Soura \\ ${ }^{5}$ Prof. \& Head, Department of CVTS, SKIMS, Soura
}

\begin{abstract}
Background: Right ventricular $(R V)$ dysfunction is a significant cause of morbidity and mortality after surgical correction of tetralogy of Fallot (TOF). Transatrial-transpulmonary repair avoids a ventriculotomy (in contrast to the transventricular approach) emphasizing maximal preservation of $R V$ structure and function. Methods: Between January 2005 to January 2014, 41 patients with TOF were referred to our unit for surgical therapy. Of these, 10 were unsuitable for repair and underwent aortopulmonary shunting. In the remaining 31 patients (mean age of $2.67 \pm 0.38$ years), complete transatrial/transpulmonary repair was performed. Previously placed shunts (four patients) were taken down. In all cases, subpulmonary resection and ventricular septal defect (VSD) closure were accomplished transatrially and transpulmonary.

Results: There were 3 (9.67\%) deaths in this series. No patient required permanent pacemaker. Median ICU and hospital stay were 91 hours and 14 days, respectively. At median follow up of 54 (mean 51 \pm 12 ) months, all patients are asymptomatic, with no significant residual lesion.

Conclusions: Transatrial/transpulmonary repair of TOF is associated with remarkably low morbidity and mortality in our early experience.
\end{abstract}

Keywords: Mortality, Morbidity, Tetralogy of fallot, Transatrial- transpulmonary repair.

\section{Introduction}

Supravalvar RVOTO seldom occurs in isolation. It may occur in tetralogy of Fallot (TOF), Williams's syndrome, Noonan syndrome, VSD, arterio-hepatic dysplasia or congenital Rubella syndrome. Tetralogy of Fallot is a cyanotic congenital cardiopathy described a century ago. La Maladie Bleue, as described by Louis Arthur Etienne Fallot in 1888 demonstrated the coexistence of four morphological abnormalities. The cardinal features were ventricular septal defect, subpulmonary stenosis, overriding aorta, and right ventricular hypertrophy ${ }^{1}$. Tetralogy of Fallot is the commonest congenital cyanotic heart disease and accounts for $5 \%$ of all congenital cardiopathies, with an incidence of about 1 in 2400 live births. ${ }^{2}$ Surgical treatment of tetralogy of Fallot (TOF) was first attempted in 1945 by Blalock and Taussig who performed a palliative subclavian-pulmonary artery shunt ${ }^{3}$. Successful repair through a right ventriculotomy was first achieved by Lillehei and Varco ${ }^{4}$, using 'controlled cross-circulation' in 1954. Traditionally, TOF was repaired through a right ventricular (RV) incision 
providing an excellent exposure for closure of the ventricular septal defect (VSD) and relief of the right ventricular outflow tract obstruction (RVOTO). Transatrial repair of RVOT obstruction in a case of TOF was first reported in 1963 by Hudspeth et al. which has been an important step in the evolution of RVOT obstruction surgery including that of TOF repair ${ }^{5}$. The benefits of the transatrial approach are believed to derive from eliminating a right ventriculotomy or uses a minimal incision onto the RV infundibulum (if annular enlargement is necessary), thus preserving the right ventricular function. The incidence of ventricular arrhythmias is less and the risk of atrial arrhythmias is not much, either.

\section{Methods}

The study was conducted from January 2005 to January 2014. In this period 41 patients with TOF (17 males, 24 females), were referred to our department for surgical management. In 10 patients, complete repair was not feasible and therefore aortopulmonary shunting was performed. The remaining 31 patients (13 males, 18 females) underwent complete repair using the transatrial-transpulmonary approach. Median body surface area (BSA) was 0.89 (mean $0.85 \pm 0.2$ ) $\mathrm{m}^{2}$. A uniform operative technique was used with bicaval cannulation for cardiopulmonary bypass and moderate systemic hypothermia $\left(28^{\circ} \mathrm{C}\right.$ nasopharyngeal temperature). Any patent shunts were taken down. Myocardial protection was achieved with intermittent cold blood cardioplegia. Via a right atriotomy and working through the tricuspid valve, the parietal extensions of the infundibular septum were divided parallel to the aortic annulus up to the level of the pulmonary valve. The dissection was completed by excision of the obstructing parietal bands, anterior infundibular trabeculations and the septal bands. The VSD was then closed transatrially using continuous prolene sutures and a tailored PTFE patch. The tricuspid valve was assessed for competence and any distortion created by the VSD patch was repaired by partial closure of the septal or antsero-septal commissure. Hegar dilators were used to assess the size of the right ventricular outflow tract (RVOT) and pulmonary valve opening and, if found to be less than mean normal according to Rowllat et al. ${ }^{6}$ in diameter, a longitudinal pulmonary arteriotomy was made. Pulmonary valvotomy was achieved by incision of the fused commissures. If required, the arteriotomy was extended via the anterior commissure through the annulus onto the RV infundibulum as long as necessary, typically $0.5-1.5 \mathrm{~cm}$, in order to achieve an RVOT diameter $2 \mathrm{~mm}$ greater than mean normal. The main pulmonary artery was augmented with an autologous pericardial patch extending across the annulus as necessary. The patch was also extended distally to repair any branch pulmonary artery hypoplasia. According to this operative protocol, VSD closure, subpulmonary resection, assessment of tricuspid valve function and tricuspid valvuloplasty, if needed, were accomplished through the right atrium in all patients. All patients underwent post-operative and before hospital discharge echocardiographic assessment of the repair. This included investigation of the presence and magnitude of any residual RVOT obstruction (RVOTO), pulmonary and/or tricuspid valve insufficiency and residual VSD, as well as assessment of overall RV and LV function.

\section{Results}

Most of our patients were of the age of 2-10 years with mean age of $2.67 \pm 0.38$ years. Out of 31 patients in our study, 18 patients were females and 13 patients were males with male: female ratio of $1: 1.38$. In our study, the mean RV/PA gradient was 82.19 with standard deviation of 10.32 and median of 80 . Majority of patients had RV/PA gradient in the range of 71-90 constituting $64.51 \%$ $(\mathrm{N}=20)$ patients. In our study, median cross clamp time was 76 minutes (mean 77.63 with S.D of 11.3 mins) with shortest and longest CCT of 60 minutes and 122 minutes, respectively. In our study, mean hospital stay was 14.77 days (median 14 days) with range of 5 to 27 days. Mean ICU stay was $94.23 \pm$ 
hours (median 91 hours). Most of patients had ICU stay ranging from 73 to 96 hours (3-4 days) constituting $70.96 \%(\mathrm{~N}=22)$ patients . In our study of 31 patients, postoperative complications occurred in 8 patients. Pulmonary complications were seen in $5(16.12 \%)$ patients, septicemia in 1 (3.22\%) patients, convulsions one patient, low output syndrome in one patient, arrhythmias in 2 $(6.45 \%)$ patients and ARF in $1(3.22 \%)$ patients. Early reoperation was needed in two patients: one patient had post operative bleeding and one patient had cardiac temponade. Echocardiography did not reveal residual VSD in any patient before discharge. The mean RVOT pressure gradient was 15.74 with a standard deviation of 6.35 . Out of 31 patients 1 patients had moderate-severe pulmonary insufficiency. Mild to moderate tricuspid valve insufficiency was seen in 1 patient and moderate impairment in RV function was seen in 1 patient.

\section{Discussion}

The surgical correction of tetralogy of Fallot can be performed with relatively low surgical mortality (0-7\%), while there is an increasing trend towards correction at a young age. Our use of transatrial-transpulmonary correction in our department is based on data from early series that reported low rates of mortality and morbidity. Our results indeed confirm that the method is very safe (low mortality), but in addition its use also contributes to the preservation of satisfactory right ventricular function, both during the early postoperative period and in the medium term. The echocardiographic data from the patients discharge examination show that only a small percentage had a moderate reduction in right ventricular contractility, while the overwhelming majority had good or very good right ventricular function. Furthermore, in this series of patients most had a small degree of pulmonary valve insufficiency. It should be stressed that the preservation of satisfactory pulmonary valve function was achieved without there being any significant degree of residual obstruction in the RVOT. In this series no patients showed a severe degree of tricuspid regurgitation during the immediate postoperative period. The early clinical and laboratory findings from this series are as follows:

- The patients remain free of symptoms, with no need for medication;

- No significant degree of RVOT obstruction has developed;

- Pulmonary valve insufficiency remains at moderate levels;

- Tricuspid valve function remains satisfactory;

- Only $2(6.45 \%)$ of patients developed arrhythmias;

- Right ventricular function remains very good in spite of the moderate pulmonary valve insufficiency.

- Out of 31 patients, $3(9.67 \%)$ died during the study period which is considered to be a low mortality rate considering that many of our patients $(17.4 \%)$ were adults. None of our patients died in the follow up period.

These results are encouraging, but the follow up must of course continue for a longer period and a more detailed study of right ventricular function will be necessary, for example using magnetic resonance imaging.

\section{Conclusion}

In conclusion, this study shows that transatrial-transpulmonary correction of the tetralogy of Fallot in our department is associated with excellent early results following surgery. Of course, further follow up is clearly necessary in order to confirm the initial findings over a longer time, with regard to the preservation of the structure and function of the right ventricle.

\section{References}

1. Anderson RH, Weinberg PM. The clinical anatomy of tetralogy of fallot. Cardiol Young. 2005;15 Suppl 1:38-47.

2. Blalock A, Taussig HB. The surgical treatment of malformations of the heart in which there is pulmonary stenosis or 
pulmonary atresia. J Am Med Assoc. 1945;128:189-92.

3. Lillehei CW, Coehn M, Warden HE, Red RC, Aust JB, De Wall RA, et al. Direct vision intracardiac surgical correction of the Tetralogy of Fallot, Pentalogy of Fallot and pulmonary atresia defects. Report of first 10 cases. Ann Surg. 1955;142 (3): 418-42.

4. Edmunds Jr LH, Saxena NG, Friedman S, Raskind WJ, Dodd PF. Transatrial repair of tetralogy of Fallot. Surg. 1976;80: 681-8.

5. Pacifico AD, Sand ME, Bargeron LM, Calvin EC. Transatrial transpulmonary repair of tetralogy of Fallot. J Thorac Cardiovasc Surg. 1987;93:919-24.

6. Rowlatt JF, Rimoldi HJ, Lev M. The quantitative anatomy of the normal child's heart, Pediatr Clin North Am. 1963;10:499-588. 